\title{
Corrigendum: Meta-analysis identifies 13 new loci associated with waist-hip ratio and reveals sexual dimorphism in the genetic basis of fat distribution
}

Iris M Heid, Anne U Jackson, Joshua C Randall, Thomas W Winkler, Lu Qi, Valgerdur Steinthorsdottir, Gudmar Thorleifsson, M Carola Zillikens, Elizabeth K Speliotes, Reedik Mägi, Tsegaselassie Workalemahu, Charles C White, Nabila Bouatia-Naji, Tamara B Harris, Sonja I Berndt, Erik Ingelsson, Cristen J Willer, Michael N Weedon, Jian’an Luan, Sailaja Vedantam, Tõnu Esko, Tuomas O Kilpeläinen, Zoltán Kutalik, Shengxu Li, Keri L Monda, Anna L Dixon, Christopher C Holmes, Lee M Kaplan, Liming Liang, Josine L Min, Miriam F Moffatt, Cliona Molony, George Nicholson, Eric E Schadt, Krina T Zondervan, Mary F Feitosa, Teresa Ferreira, Hana Lango Allen, Robert J Weyant, Eleanor Wheeler, Andrew R Wood, MAGIC, Karol Estrada, Michael E Goddard, Guillaume Lettre, Massimo Mangino, Dale R Nyholt, Shaun Purcell, Albert Vernon Smith, Peter M Visscher, Jian Yang, Steven A McCarroll, James Nemesh, Benjamin F Voight, Devin Absher, Najaf Amin, Thor Aspelund, Lachlan Coin, Nicole L Glazer, Caroline Hayward, Nancy L Heard-Costa, Jouke-Jan Hottenga, Åsa Johansson, Toby Johnson, Marika Kaakinen, Karen Kapur, Shamika Ketkar, Joshua W Knowles, Peter Kraft, Aldi T Kraja, Claudia Lamina, Michael F Leitzmann, Barbara McKnight, Andrew P Morris, Ken K Ong, John R B Perry, Marjolein J Peters, Ozren Polasek, Inga Prokopenko, Nigel W Rayner, Samuli Ripatti, Fernando Rivadeneira, Neil R Robertson, Serena Sanna, Ulla Sovio, Ida Surakka, Alexander Teumer, Sophie van Wingerden, Veronique Vitart, Jing Hua Zhao, Christine Cavalcanti-Proença, Peter S Chines, Eva Fisher, Jennifer R Kulzer, Cecile Lecoeur, Narisu Narisu, Camilla Sandholt, Laura J Scott, Kaisa Silander, Klaus Stark, Mari-Liis Tammesoo, Tanya M Teslovich, Nicholas John Timpson, Richard M Watanabe, Ryan Welch, Daniel I Chasman, Matthew N Cooper, John-Olov Jansson, Johannes Kettunen, Robert W Lawrence, Niina Pellikka, Markus Perola, Liesbeth Vandenput, Helene Alavere, Peter Almgren, Larry D Atwood, Amanda J Bennett, Reiner Biffar, Lori L Bonnycastle, Stefan R Bornstein, Thomas A Buchanan, Harry Campbell, Ian N M Day, Mariano Dei, Marcus Dörr, Paul Elliott, Michael R Erdos, Johan G Eriksson, Nelson B Freimer, Mao Fu, Stefan Gaget, Eco J C Geus, Anette P Gjesing, Harald Grallert, Jürgen Gräßler, Christopher J Groves, Candace Guiducci, Anna-Liisa Hartikainen, Neelam Hassanali, Aki S Havulinna, Karl-Heinz Herzig, Andrew A Hicks, Jennie Hui, Wilmar Igl, Pekka Jousilahti, Antti Jula, Eero Kajantie, Leena Kinnunen, Ivana Kolcic, Seppo Koskinen, Peter Kovacs, Heyo K Kroemer, Vjekoslav Krzelj, Johanna Kuusisto, Kirsti Kvaloy, Jaana Laitinen, Olivier Lantieri, G Mark Lathrop, Marja-Liisa Lokki, Robert N Luben, Barbara Ludwig, Wendy L McArdle, Anne McCarthy, Mario A Morken, Mari Nelis, Matt J Neville, Guillaume Paré, Alex N Parker, John F Peden, Irene Pichler, Kirsi H Pietiläinen, Carl G P Platou, Anneli Pouta, Martin Ridderstråle, Nilesh J Samani, Jouko Saramies, Juha Sinisalo, Jan H Smit, Rona J Strawbridge, Heather M Stringham, Amy J Swift, Maris Teder-Laving, Brian Thomson, Gianluca Usala, Joyce B J van Meurs, Gert-Jan van Ommen, Vincent Vatin, Claudia B Volpato, Henri Wallaschofski, G Bragi Walters, Elisabeth Widen, Sarah H Wild, Gonneke Willemsen, Daniel R Witte, Lina Zgaga, Paavo Zitting, John P Beilby, Alan L James, Mika Kähönen, Terho Lehtimäki, Markku S Nieminen, Claes Ohlsson, Lyle J Palmer, Olli Raitakari, Paul M Ridker, Michael Stumvoll, Anke Tönjes, Jorma Viikari, Beverley Balkau, Yoav Ben-Shlomo, Richard N Bergman, Heiner Boeing, George Davey Smith, Shah Ebrahim, Philippe Froguel, Torben Hansen, Christian Hengstenberg, Kristian Hveem, Bo Isomaa, Torben Jørgensen, Fredrik Karpe, Kay-Tee Khaw, Markku Laakso, Debbie A Lawlor, Michel Marre, Thomas Meitinger, Andres Metspalu, Kristian Midthjell, Oluf Pedersen, Veikko Salomaa, Peter E H Schwarz, Tiinamaija Tuomi, Jaakko Tuomilehto, Timo T Valle, Nicholas J Wareham, Alice M Arnold, Jacques S Beckmann, Sven Bergmann, Eric Boerwinkle, Dorret I Boomsma, Mark J Caulfield, Francis S Collins, Gudny Eiriksdottir, Vilmundur Gudnason, Ulf Gyllensten, Anders Hamsten, Andrew T Hattersley, Albert Hofman, Frank B Hu, Thomas Illig, Carlos Iribarren, Marjo-Riitta Jarvelin, W H Linda Kao, Jaakko Kaprio, Lenore J Launer, Patricia B Munroe, Ben Oostra, Brenda W Penninx, Peter P Pramstaller, Bruce M Psaty, Thomas Quertermous, Aila Rissanen, Igor Rudan, Alan R Shuldiner, Nicole Soranzo, Timothy D Spector, Ann-Christine Syvanen, Manuela Uda, André Uitterlinden, Henry Völzke, Peter Vollenweider, James F Wilson, Jacqueline C Witteman, Alan F Wright, Gonçalo R Abecasis, Michael Boehnke, Ingrid B Borecki, Panos Deloukas, Timothy M Frayling, Leif C Groop, Talin Haritunians, David J Hunter, Robert C Kaplan, Kari E North, Jeffrey R O’Connell, Leena Peltonen, David Schlessinger, David P Strachan, Joel N Hirschhorn, Themistocles L Assimes, H-Erich Wichmann, Unnur Thorsteinsdottir, Cornelia M van Duijn, Kari Stefansson, L Adrienne Cupples, Ruth J F Loos, Inês Barroso, Mark I McCarthy, Caroline S Fox, Karen L Mohlke \& Cecilia M Lindgren Nat. Genet. 42, 949-960 (2010); published online 10 October 2010; corrected after print 12 October 2011

In the version of this article initially published, there were errors in Table 1. Specifically, for eight SNPs, the effect allele frequencies were reversed. The correct effect allele frequencies for rs9491696, rs984222, rs4846567, rs1011731, rs718314, rs1294421, rs6795735 and rs2076529 are 0.480, $0.635,0.717,0.428,0.259,0.613,0.594$ and 0.430 , respectively. These errors have been corrected in the HTML and PDF versions of the article.

\section{Corrigendum: Genome-wide association study identifies 12 new susceptibility loci for primary biliary cirrhosis}

George F Mells, James A B Floyd, Katherine I Morley, Heather J Cordell, Christopher S Franklin, So-Youn Shin, Michael A Heneghan, James M Neuberger, Peter T Donaldson, Darren B Day, Samantha J Ducker, Agnes W Muriithi, Elizabeth F Wheater, Christopher J Hammond, Muhammad F Dawwas, The UK PBC Consortium, The Wellcome Trust Case Control Consortium 3, David E Jones, Leena Peltonen, Graeme J Alexander, Richard N Sandford \& Carl A Anderson Nat. Genet. 43, 329-332 (2011); published online 13 March 2011; corrected online 12 October 2011

In the version of this article initially published, three authors, Paul Richardson, Ikram Nasr and Richard Aspinall, were inadvertently omitted from the list of the members of the UK PBC Consortium provided in the Supplementary Note. The error has been corrected in the supplementary information file. 